\title{
Prediction of Primary Atomization using Smoothed Particle Hydrodynamics
}

\author{
Rainer Koch*, Samuel Braun, Lars Wieth, Geoffroy Chaussonnet, Thilo Dauch, Hans-Jörg Bauer
}

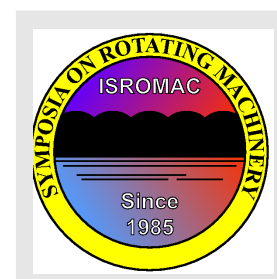

ISROMAC 2016

International

Symposium on Transport

Phenomena and

Dynamics of

Rotating

Machinery

Hawaii, Honolulu

April 10-15, 2016

\begin{abstract}
In modern jet engines mostly air blast atomizers are used for the liquid fuel injection. The prediction of the spray generated by such atomizers was for a long time not feasible because of restricted computing resources. However, with modern super-computers the prediction of the atomization has come into reach.
\end{abstract}

In the present paper a new approach for the numerical prediction of the primary atomization is presented. The methodology is based on the Smoothed Particle Hydrodynamics (SPH), which has originally been developed in the context of astrophysics.

The numerical predictions to be presented were performed for a planar model atomizer, for which a vast amount of experimental data was collected by us previously. The major objectives of the numerical predictions are to elaborate the mechanism governing the effect of thickness of the trailing edge of the prefilmer on the size of the droplets and the temporal droplet formation rate.

\section{Keywords}

Fuel injection - Atomization - Numerical prediction — Smoothed Particle Hydrodynamics

\section{INTRODUCTION}

In civil aviation, fuel injection is one of the most crucial processes affecting engine emissions, in particular soot and NOx emissions. First experimental investigations into air blast atomization date back to the 70's 11. But even today, the basic mechanism governing air blast atomization is not understood, and reliable modelling approaches are not available. Present state of the art is to empirically optimize the fuel injectors. Typically, expensive measurements of the injector performance in terms of droplet size and velocity are performed at elevated pressures. Afterwards correlations like those proposed by e.g. Lefebvre [2, 3] are used to extrapolate the experimental results to other operating conditions.

The prediction of the atomization process based on first principles was for a long time not feasible because of restricted computing resources. However, with modern super-computers providing 10.000 or even more CPUs, such predictions have come into reach. But the prediction of the atomization process is still a major challenge, as a wide range of scales in space and time must be captured. The spatial range extends from $1 \mu \mathrm{m}$ which resembles the typical dimensions of the thickness of the bubbles formed during the disintegration process up to the distance required for finalizing secondary break up of larger droplets or ligaments, which is in the range of approximately $0.1 \mathrm{~m}$. Time scales reach from $1 \mu \mathrm{s}$ for the typical interaction between the air flow and the liquid at the trailing edge up to the residence time inside the combustor of typically $10 \mathrm{~ms}$.

The Institut für Thermische Strömungsmaschinen (ITS) has started 7 years ago to develop a numerical method for predicting the primary atomization of air blast nozzles, which are typically used in jet engines. The numerical approach is based on the mesh free Smoothed Particle Hydrodynamics (SPH) method [4, which has originally been developed in the context of astrophysics.

The SPH method was chosen because it has some inherent advantages over the readily available and commonly used grid based methods such as the Volume of Fluid (VoF) method. Recent developments of applying the VoF method for predicting atomization are discussed by Hermann [5].

During primary atomization the liquid surface is exposed to large deformations, and surface tension plays a crucial role for the liquid disintegration. Because of its Lagrangian nature, the Smoothed Particle Hydrodynamics (SPH) method adapts itself naturally to large deformations of the gas liquid interface. Furthermore, the curvature of the interface, which is required for computing the surface tension, can directly be calculated and does not require a cumbersome surface reconstruction technique like in all grid based methods.

In the present paper the application of the SPH method for predicting primary atomization will be presented and discussed. All predictions are reflecting an experiment which was performed at a generic planar prefilming air- 
blast atomizer. The experimental setup and results have been published at several occasions [6, 7, 8].

The paper is organized as follows: First the fundamentals of the SPH method will be introduced. Then, a summary of the experimental study of the planar air blast atomizer will be given. The experimental results are predicted using the SPH method. Special emphasis is put on the effect of the thickness of the trailing edge of the prefilmer on the atomization. These predicted results are compared in terms of droplet size distribution, ligament lengths and breakup frequencies to experimental data.

\section{SMOOTHED PARTICLE HYDRO- DYNAMICS METHOD}

The particle based SPH method has been developed in the late 1970's in the context of astrophysics [4. Recently, it gained popularity in general computational fluid dynamics, where the main scope is on the simulation of free surface flows, e.g. 9]. In contrast to commonly used grid based Eulerian methods, the spatial discretization of a computational domain is performed via so called particles, which represent a small volume of the fluid. Due to the Lagrangian nature of the method, the particles move within the computational domain with the actual fluid velocity. The governing equations describing the flow physics are the Navier Stokes equations.

The main idea behind the SPH formalism is to evaluate the physical property of a particle or its spatial derivative by interpolating over neighbor particles within a certain radius of influence. Equation (11) reflects the basic interpolation formalism for a particle with index $i$.

$$
\langle\Phi\rangle_{i}=\sum_{j} V_{j} \Phi_{j} W\left(\vec{x}_{i}-\vec{x}_{j}, h\right)
$$

In Eq. (1), $\Phi$ is a physical quantity which may be e.g. the density or the velocity etc. $V_{j}$ is the volume of an adjacent particle $j$ and $W\left(\vec{x}_{i}-\vec{x}_{j}, h\right)$ is a weighting function, which depends on the positions $\vec{x}_{i / j}$ and the smoothing length $h$. The summation is truncated once the distance to the adjacent particle exceeds the predefined limit $\Delta r$, which depends on the smoothing length. For a more detailed description of the SPH basics, please refer to e.g. 10. The interpolation procedure is illustrated in Fig 1.

Until now, the application of SPH to technical problems was limited by the lack of suitable boundary conditions. Recently, flexible and robust inflow and outflow boundaries have been introduced and been demonstrated to be successfully incorporated into a massively parallel framework 11. In contrast to our previous work [10], realistic boundary conditions and fluid properties are now available for predictions of technically relevant flow situations.

The modeling of surface tension plays a crucial role when predicting the atomization process. This is due

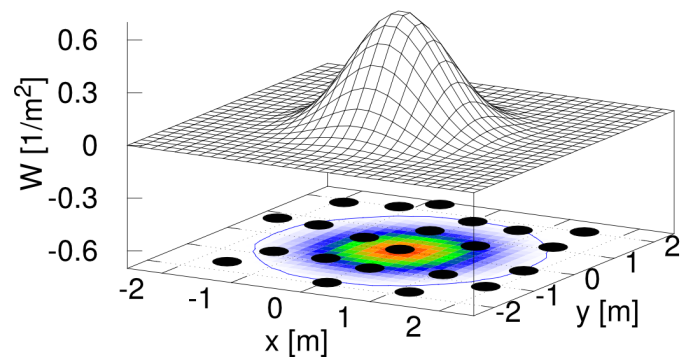

Figure 1. Principle of the interpolation within the SPH Method

to the fact, that the liquid disintegration is determined by the balance of two forces acting at the liquid-air interface: The surface tension forces and the shear forces induced by the air flow. In our SPH code the surface tension is represented by the Continuum Surface Force (CSF) model, which was originally introduced by Brackbill et al. 12] in the context of the VoF method. The CSF model as adopted in our approach was proposed by Adami et al. 13. The key feature of the CSF model is the representation of the surface tension force as continuous force acting on the volume adjacent to the interface instead of the surface of the interface between liquid and air.

Wetting effects, which may significantly affect primary atomization, are accounted for by the model of Wieth et al. 14. In order to cope with the high density ratios of up to 1000 depending on the ambient air pressure, the continuity equation as proposed by $\mathrm{Hu}$ et al. [15] is applied

$$
\langle\rho\rangle_{i}=m_{i} \sum_{j} W\left(\vec{x}_{i}-\vec{x}_{j}, h\right)
$$

where $m_{i}$ is the mass of the center particle.

\section{EXPERIMENTAL STUDY OF AIR BLAST ATOMIZATION}

\subsection{Experimental setup and diagnostics}

For studying experimentally the liquid disintegration of air blast atomizers, and also for the validation of predictions, a generic experiment resembling the characteristics of a typical air blast atomizer was set up. A planar atomizer geometry was used instead of the annular design of real fuel injector nozzles [16]. This planar geometry enables better access for optical diagnostics. A sketch of the atomizer is shown in Fig. 2.

On top of the planar prefilmer plate, a kerosene surrogate is supplied. The liquid disintegration takes place downstream at the trailing edge of the prefilmer. In Fig. 3 a top view on the trailing edge of the prefilmer is depicted. The flow direction is from top to bottom. The photograph (a) was taken at low air velocity, photograph (b) at high air velocity. It is clearly visible that 


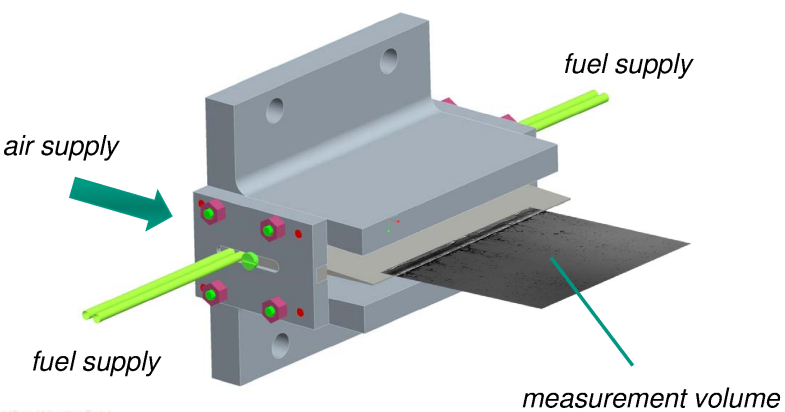

Figure 2. Generic planar air blast atomizer

the liquid is accumulated at the trailing edge of the prefilmer lip. The accumulated liquid is periodically deformed into bubbles. In Fig. 4 a zoom into the region at the trailing edge is depicted, revealing the formation of such bubbles. Once a bubble bursts, lengthy ligament structures are formed, which are periodically torn off the trailing edge.

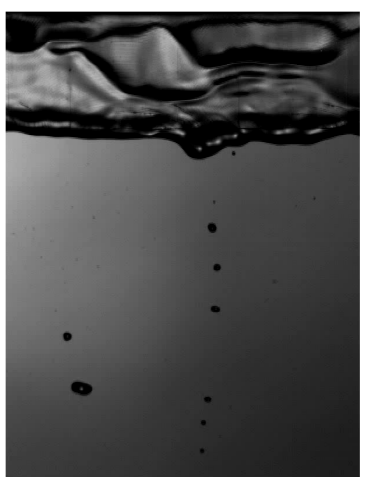

(a)

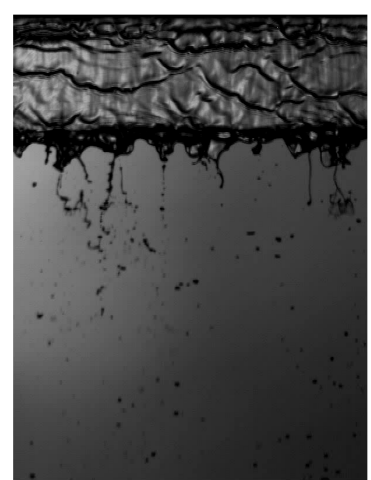

(b)
Figure 3. Film flow and liquid disintegration at the trailing edge of the prefilmer: (a) low air velocity; (b) high air velocity

Numeral series of atomization experiments have been performed using liquids of different surface tension and viscosity. In addition to varying the air speed and liquid loading, also different operating pressures have been looked at. The details of the experimental setup and the results are documented in several publications [6, 7, 8]. Here it is sufficient to say that a shadowgraphy setup was used in conjunction with a high speed camera taking frames at $13.000 \mathrm{~Hz}$. The excellent optical accessibility enables a high spatial resolution for the high-speed video recordings. The typical spatial dimension of such a frame was $15 \mathrm{~mm} \times 20 \mathrm{~mm}$, resulting in a spatial resolution of approximately $10 \mu \mathrm{m}$ per pixel. These high speed recordings were then post-processed in order to extract the desired data. The absence of a spanwise cross flow and of secondary vortices facilitates the separation and identification of physical processes affecting

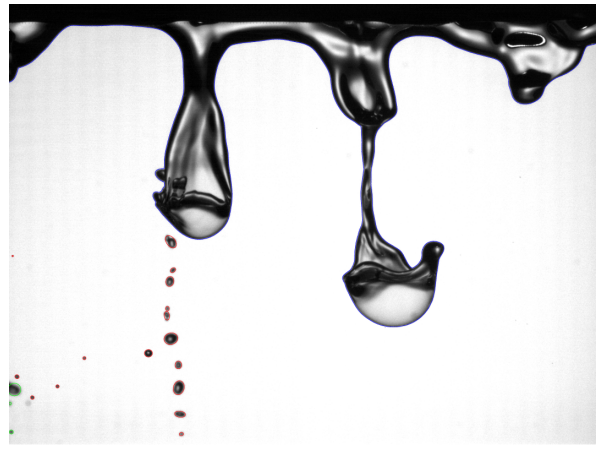

Figure 4. Bubble formation during liquid disintegration

liquid atomization.

\subsection{Experimental results}

One of the most striking results of the experimental study was the finding that the thickness of the trailing edge does affect the atomization process, as long as the edge is thicker than the liquid film. In particular it was found that the droplet generation frequency decreases and the size of the droplet increases when the trailing edge becomes thicker [6]. These results are plotted in Fig. 5 .

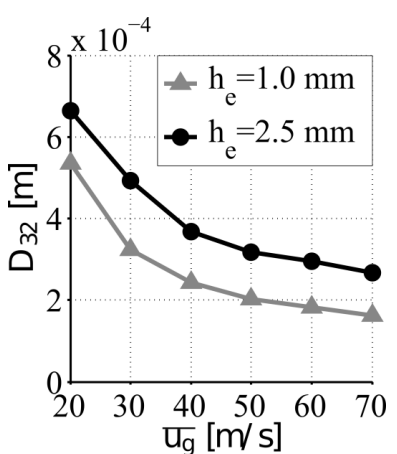

(a)

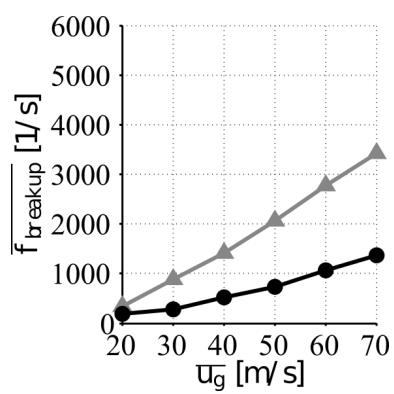

(b)
Figure 5. Effect of trailing edge thickness on droplet size (a) and detachment frequency (b)

Gepperth et al. 6] argued, that the effect of the trailing edge thickness is mainly due to the liquid accumulation at the trailing edge. The volume of the accumulated liquid depends on the trailing edge height, because its thickness is governed by the edge height. At the end the accumulated liquid volume determines the volume of the ligaments to be torn off, and thus the droplet size. At a given liquid film flow rate, the frequency of the detachment of the ligament is inversely proportional to the volume of the ligaments generated. Therefore, at a large edge height, bigger droplets are observed which detach at lower frequency.

In deed, the data of Gepperth showed that the mean breakup frequency $\overline{f_{\text {breakup }}}$ is inversely proportional to 
the trailing edge height $h$.

$$
\operatorname{Sr}_{f, \text { breakup }} \sim h \cdot \overline{f_{\text {breakup }}}
$$

In Eq. (3), the dimensionless breakup frequency is expressed in terms of the Strouhal number $\mathrm{Sr}_{f \text {, breakup. More- }}$ over, Gepperth could derive a relation between the Sauter mean diameter (SMD) of the fuel spray and the trailing edge height:

$$
\mathrm{SMD} \sim h^{0.46}
$$

Another interesting observation could also be confirmed by the experiments of Gepperth et al.: The film waves arriving at the trailing edge do not affect the liquid disintegration process. The wavy film causes temporal and spatial fluctuations of liquid supply to the trailing edge. But these fluctuations are suppressed by the accumulation of the liquid at the trailing edge. Therefore, the film waves do not have any impact on the frequency of the ligament formation. This mechanism has been reported approximately 30 years ago by Sattelmayer and Wittig [1], who investigated a prefilmer with an edge thickness of $h \approx 200 \mu \mathrm{m}$.

In contradiction to the findings of Gepperth, there is another quite similar experimental study by Inamura et al. 17. The authors could not identify any effect of the trailing edge height neither on droplet size nor on the break up frequency. In contrast to the setup of Gepperth, Inamura et al. used a rather sharp edged prefilmer lip $(h \approx 160 \mu \mathrm{m}[18$ ). The designs used by Gepperth had rather thick trailing edges of $230 \mu \mathrm{m}, 1.0 \mathrm{~mm}$ and $2.5 \mathrm{~mm}$.

Despite some sophisticated experimental setups, up to now the role of the prefilmer edge height in the atomization process is not understood. In this context it has to be considered, that the interaction of film waves with the accumulation of the liquid at the trailing edge makes it difficult to properly discriminate the phenomena involved and their impact on the atomization performance from experiments. As consequence, up to now no correlation did exist which includes the effect of the trailing edge thickness on droplet size and frequency. The only correlation accounting for this effect is the one proposed recently by Gepperth [7] as expressed by Eqs. [3. 41). However, presently it is not clear within which range those correlations are valid. In particular it is of major interest, to clarify, why there seems to be no effect at rather thin trailing edges.

It is the objective of this paper to present numerical simulations of the atomization process based on the SPH method, which will serve to clarify this effect.

\section{NUMERICAL SETUP FOR THE SPH PREDICTIONS}

The numerical results to be presented subsequently were produced by considering a $2 \mathrm{D}$ computational domain, which represents the trailing edge of the prefilmer. The numerical domain covers just the close vicinity of the trailing edge as illustrated in Fig. 6.
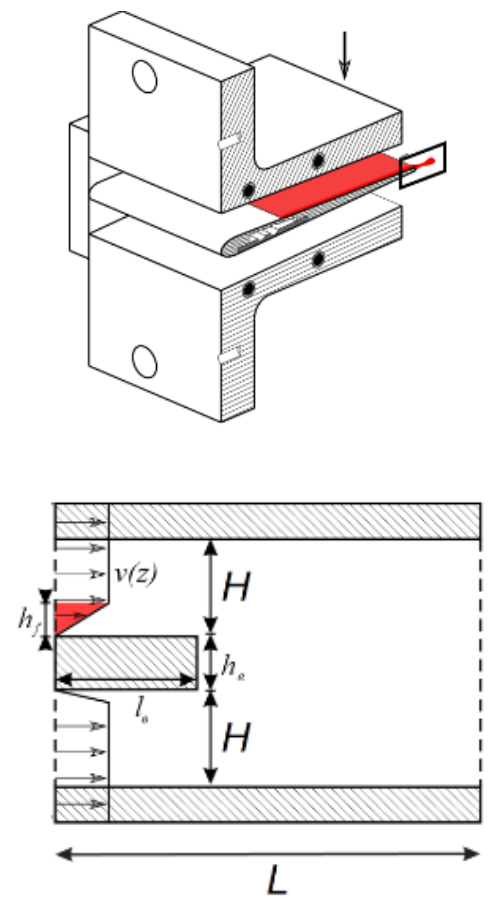

Figure 6. Setup of the planar air blast atomizer for the numerical predictions

\begin{tabular}{llcl}
\hline Geometry & & & \\
\hline Length of the domain & $\mathrm{L}$ & 6 & {$[\mathrm{~mm}]$} \\
Length of the prefilmer & $\mathrm{l}_{\mathrm{e}}$ & 2 & {$[\mathrm{~mm}]$} \\
Height of Air flow channels & $\mathrm{H}$ & 3 & {$[\mathrm{~mm}]$} \\
Thickness of trailing edge & $\mathrm{h}_{\mathrm{e}}$ & $25,50,100$, & \\
& & 230,400 & {$[\mu \mathrm{m}]$} \\
Thickness of the liquid film & $\mathrm{h}_{\mathrm{f}}$ & 80 & {$[\mu \mathrm{m}]$} \\
Angle of the prefilmer & $\alpha$ & 4.29 & {$\left[{ }^{\circ}\right]$} \\
Spatial resolution & $\mathrm{dx}$ & 5 & {$[\mu \mathrm{m}]$} \\
\hline
\end{tabular}

Table 1. Geometrical dimensions of the numerical domain

The computational domain comprises two inflow boundaries at the left, an outlet boundary at the right. The air enters the domain at the left with a piecewise linear velocity profile, which is intended to mimic a boundary layer close to the walls. At the boundaries at the top and at the bottom of the computational domain, the velocity in axial direction is imposed, which is set to the free stream velocity. This is achieved by imposing walls moving with the free stream velocity at the top and the bottom of the domain. These wall are represented as hatched areas in Fig(1). The domain includes 
the prefilmer lip. Here, the air velocity is set equal to the film velocity. The liquid film enters at the inflow boundary with a block shaped velocity profile. The liquid loading $\dot{V}$ corresponds to an experimentally examined operating point. The variation of the trailing edge height only affects $\mathrm{h}_{\text {trailing edge }}$ and the total height of the domain. The inflow channel height $h_{\text {inlet }}$ remains unaltered. Therefore, the flow regimes for all investigated trailing edges are assumed to be identical. Geometrical details, the operating conditions and the fluid properties are listed in Tabs. 1 and 2 .

\begin{tabular}{|c|c|c|c|}
\hline \multicolumn{4}{|c|}{ Operating conditions and fluid properties } \\
\hline & Air & Liquid & \\
\hline Inlet velocity & $0-50$ & 0.617 & {$\left[\mathrm{~m} \mathrm{~s}^{-1}\right]$} \\
\hline Operating pressure & \multicolumn{2}{|c|}{1} & [bar] \\
\hline$\rho$ & 1 & 770 & {$\left[\mathrm{~kg} \mathrm{~m}^{-3}\right]$} \\
\hline$\mu$ & $1.8 \mathrm{e}-5$ & $1.56 \mathrm{e}-3$ & {$[\mathrm{Pas}]$} \\
\hline$\sigma_{\text {air-liquid }}$ & \multicolumn{2}{|c|}{0.0275} & {$\left[\mathrm{Nm}^{-1}\right]$} \\
\hline Contact angle $_{\text {wall-liquid }}$ & \multicolumn{2}{|c|}{60} & {$\left[{ }^{\circ}\right]$} \\
\hline
\end{tabular}

Table 2. Inlet conditions and fluid properties

In order to determine a suitable spatial resolution, preliminary simulations have been performed with a mean particle spacing $d x$ of $20 \mu \mathrm{m}, 10 \mu \mathrm{m}, 5 \mu \mathrm{m}$ and $2.5 \mu \mathrm{m}$. The effect of the spatial resolution is detailed and discussed on computational performance and accuracy is discussed in a complementary paper [19]. This paper contains also a comparison of the computational performance of the SPH method in comparison to the Volume of Fluid method as available in OpenFOAM package as well as in FLUENT package.

Concerning the gaseous flow, a resolution of $d x=$ $10 \mu \mathrm{m}$ was found to be sufficient. A further refinement did not alter the flow field. The liquid phase, however, requires a much finer spatial resolution. An indicator for the necessity of a spatial discretization refinement is the occurrence of single particle stripping. If the spatial discretization is too coarse, single particles are torn off from the ligaments or droplets, which are exposed to strong shear flows. Even at a resolution of $d x=2.5 \mu \mathrm{m}$, single particle stripping could be observed. However, the portion of single particle droplets (which can not be considered as physical droplets) strongly decreases when reducing the spatial resolution from $10 \mu \mathrm{m}$ to $5 \mu \mathrm{m}$.

Taking into account the computational effort of the simulations, a mean particle spacing of $d x=5 \mu \mathrm{m}$ has been chosen as a reasonable compromise for the present study. Thus, the computational domain consists of approximately 1.5 million particles. 4 million time steps ( $23 \mathrm{~ms}$ of physical time) have been performed in approximately 18 hours wall-clock time on 900 CPUs on the high performance parallel computer cluster ForHLR 11 .

\footnotetext{
${ }^{1}$ Forschungshochleistungsrechner ForHLR (Phase) I
}

Furthermore, it has to be emphasized that no turbulence models have been used for the current predictions. Because of the fine spatial resolution of approximately $d x=5 \mu \mathrm{m}$, most of the turbulent length scales are captured and resolved directly, i.e. virtually a direct numerical simulation (DNS) is performed. At the present stage of development of the SPH no methodology for generating artificial, synthetic turbulence is available. Therefore, in the present study no turbulent fluctuations have been imposed at the inlet of the duct. However, the authors are aware that this issue needs further research in the future.

\section{POST PROCESSING OF THE NUMERI- CAL RESULTS}

One major goal of the present study was to extract the dimensions, the volume and the velocity of the ligaments and droplets formed during the break up process. In order to identify theses quantities, a post processing technique was developed with the goal to properly detect ligaments and droplets.

In this context, a ligament or droplet is defined as a connected cluster of SPH-particles consisting of liquid. Adjacent SPH-particles, which are of the 'liquid' type, are assumed to form a ligament or droplet, if the interparticle distance is smaller than the kernel radius $\Delta r$. A special cluster detection algorithm has been developed, which incorporates a kd-tree neighbor search and an iterative cluster propagation technique. After the detection step, a unique ID is assigned to each cluster. This enables to classify each cluster by e.g. the center of gravity and its velocity or by its sphericity. Due to the known number of SPH-particles contained within each cluster, the mass of the ligament or droplet is known without any additional assumptions. Based on the mass of a cluster, a representative droplet diameter is calculated by assuming a spherical shape.

By considering all detected droplets and ligaments within the entire computational domain for each time step, the temporal evolution of the characteristic diameters and the numbers of droplets can be obtained. The temporal resolution of the numerical prediction is approximately $11.2 \mu \mathrm{s}$ or $89.5 \mathrm{kHz}$. Due to this high sampling rate, droplets within the computational domain are detected multiple times. This approach of analyzing the data is identical to that which was used for the postprocessing of the experimental data, where the sampling rate is also very high at approximately $10 \mathrm{kHz}[20$.

If all single ligaments and droplets are properly detected, derived quantities can be computed. For example, the volumetric diameters $D_{\mathrm{V} 0.1}, D_{\mathrm{V} 0.5}$ and $D_{\mathrm{V} 0.9}$ were calculated by sorting the droplet volumes and by

http://www.top500.org/system/178424

http://www.bwhpc-c5.de/wiki/index.php/ForHLR_Phase_I_-_Ha rdware_and_Architecture 
calculating the accumulated volume distribution. Knowing the density of the liquid and the droplet mass, the volumetric diameters have been determined by converting the corresponding percentile volumes.

Typical results which were derived on basis of this post-processing technique will be discussed in sec. 5.4 and 5.5

\section{RESULTS AND DISCUSSION}

\subsection{Limitations of the numerical predictions}

Despite the fact that for the present numerical predictions leading edge high performance computers were used, there are still limitations of the present numerical study. One issue is that the geometrical dimensions of the computational domain is very confined. The length in streamwise direction is definitely too small for a correct representation of the liquid break up process. The limited length of the prefilmer in stream-wise direction of just $2 \mathrm{~mm}$ will not allow a correct evolution of the liquid film flow, in particular the build up of the waves on top of the film. This is not a real problem, because there is experimental evidence that the wave structure of the film will not affect the frequency of the break up nor the volume of the ligaments, because the film waves and break up process are decoupled [1, 6].

The second issue is the limited statistics of the numerical simulations. In order to keep the computational effort treatable, only a few, typically 20 breakup events can be simulated. This is by far not sufficient for a sound comparison to the experiment. A third shortcoming is also due to the limitation of the computational effort: As stated in sec. 3. the spatial discretization selected for the present predictions is $5 \mu \mathrm{m}$. This is not enough to resolve the bubbles which are formed during liquid disintegration.

The most severe limitation is imposed by the fact that all simulations are two-dimensional. It is well known from experiments, that the breakup process is governed by instabilities of the liquid in span-wise direction along the trailing edge [6]. The instabilities in span-wise direction trigger the formation of the bubbles as shown in Fig,4. These instabilities can of course not be captured by the present $2 \mathrm{D}$ setup. Consequently, the exact representation of the bubble formation is beyond of the scope of the present simulations.

\subsection{Objective of the numerical predictions}

The hypothesis to be elucidated by the present simulations is that the accumulation of liquid at the trailing edge may be the cause for the experimental observations that the thickness does affect the break up frequency and the size of the droplets. In particular it is to be clarified, if the relation between the thickness of the trailing edge and the droplet size (Eq,4), as it was found from the experiments can be confirmed by the numerical simulations.
The build up of the liquid reservoir along the trailing edge is supposed to be the most important effect governing droplet size and droplet detachment frequency. This process will be well captured by the present $2 \mathrm{D}$ simulations. Therefore, the present numerical setup is considered to be suitable for validating this hypothesis.

\subsection{Liquid accumulation and break up}

Snapshots showing the liquid accumulation and the formation of the ligaments at the trailing edge are compiled in Fig. 7.

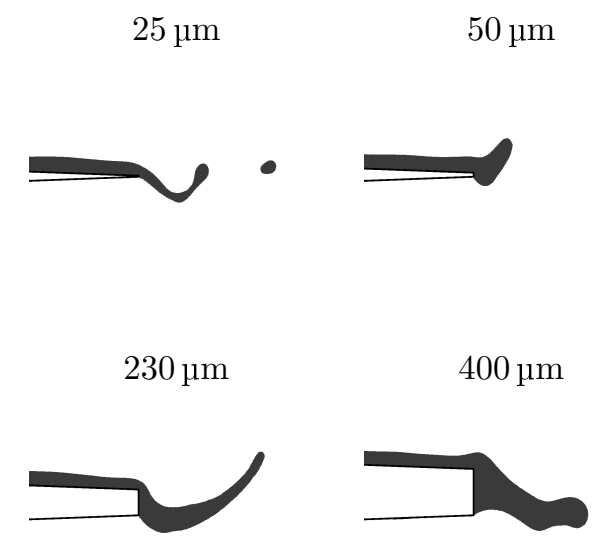

Figure 7. Effect of trailing edge height on ligament formation and liquid accumulation

In this Figure the scenario is displayed for different thicknesses of the trailing edge. It is clearly visible that there is an accumulation of liquid at the end of the trailing edge. For the cases of $230 \mu \mathrm{m}$ and $400 \mu \mathrm{m}$ edge thickness, the accumulated volume of liquid clearly depends on the thickness of the edge. In the case of $25 \mu \mathrm{m}$ no accumulation is visible. These observations support the experimental findings that the diameter of the droplets will increase with the increasing thickness of the edge for a thick trailing edge, i.e. $h_{E d g e} \geq 230 \mu \mathrm{m}$. Moreover, since there is no accumulation in the case of $25 \mathrm{\mu m}$ edge thickness, the droplet size cannot be affected by the same mechanism.

The break up process and the subsequent ligament formation is depicted in Fig. 8 for the cases of a thin $(25 \mu \mathrm{m})$ and a thick trailing edge $(230 \mu \mathrm{m})$. The time span between two consecutive images $\Delta t$ is approximately $134 \mathrm{\mu s}$. The unsteady air flow in the wake of the prefilmer exhibits periodic oscillations which trigger the flapping of the ligaments, as long as those are attached to the trailing edge. Due to the shear forces imposed to the ligament, these are elongated and finally disintegrated. Even from these 2D simulations one can identify a shape which resembles the bag like structures that 


$$
25 \mu \mathrm{m}
$$
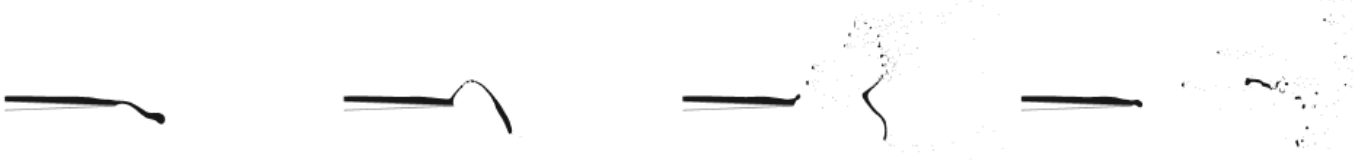

$$
230 \mu \mathrm{m}
$$
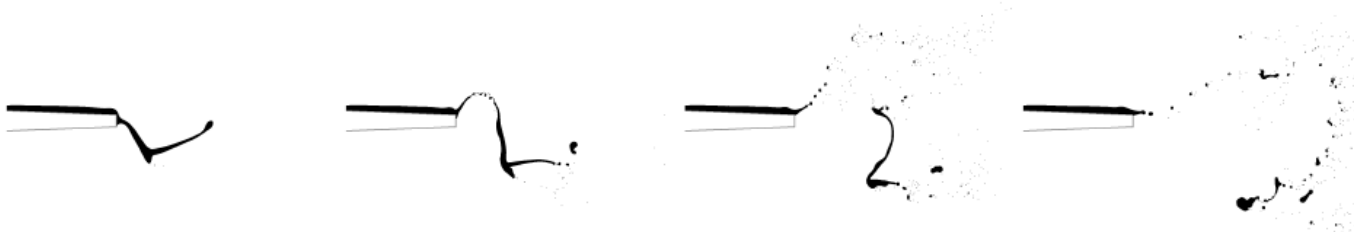

Figure 8. Time series of an arbitrary breakup event for a thin trailing edge height of $25 \mu \mathrm{m}$ (top) and a thick one of $230 \mu \mathrm{m}$ (bottom)

were observed in the experiment, e.g. in the third snapshot in both rows. Obviously, a part of the primary ligament is deformed into the bag like structure which is immediately destroyed, and the other part of the ligament survives this first break up event. It will be disintegrated later on downstream the trailing edge by the secondary break up mechanism.

The obvious difference between the thin and the thick trailing edge case is that the ligaments that survive the first break up are thicker and longer in case of the thicker trailing edge. Thus, these ligaments contain a higher volume. This may partly explain, why the resulting droplets are bigger in case of the thick trailing edge.

\subsection{Temporal evolution of ligament elonga- tion and droplet formation}

Based on the post processing method which was explained in sec. 4, the numerical results have been further analyzed. The goal was to extract time series of the ligament elongation and the droplet formation. The definition of the ligament length as used subsequently is illustrated in Fig. 9.

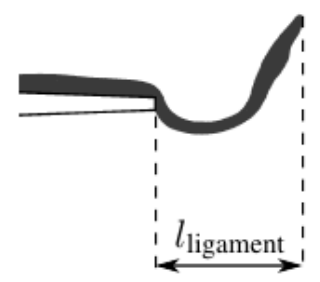

Figure 9. Definition of ligament length

The break up length was determined from the length of the ligament just at the moment where the ligament had reached its maximum length, i.e. maximum extension downstream.

After the ligament is detached from the trailing edge, the calculated ligament length will drop suddenly, because a new ligament will be formed. The time scale between two sudden drops of the ligaments length was used to define the breakup frequency.

Typical time series of the ligament length and the number of droplets generated are plotted in Fig. 10 for different thicknesses of the trailing edge.

In general, the evolution of the ligament length is characterized by a sawtooth shaped curve. As mentioned previously, the primary breakup event is characterized by an abrupt drop of this curve. Simultaneously with the sudden drop of the ligament length, the number of droplets increases. The curve representing the number of droplets is characterized by triangular shape. The ascending branch of the triangles indicates the generation of droplets. Obviously, droplet generation takes place immediately after the detachment of the ligament. This process can be identified as primary atomization. An example is highlighted by "primary" in Fig. 10. This disintegration of the ligaments into droplets, which causes the strong increase of the number of droplets is illustrated by the two most right frames in Fig. 8 .

But also secondary atomization can observed in the time series in Fig. 10. Secondary atomization means that larger droplets or ligaments which are detached from the trailing edge undergo further disintegration. Such an example is marked as "secondary" in Fig. 10.

The temporal evolution of the $25 \mu \mathrm{m}$ and $50 \mu \mathrm{m}$ case is very similar: The maximum ligament length (breakup length) as well as the number of generated droplets are 


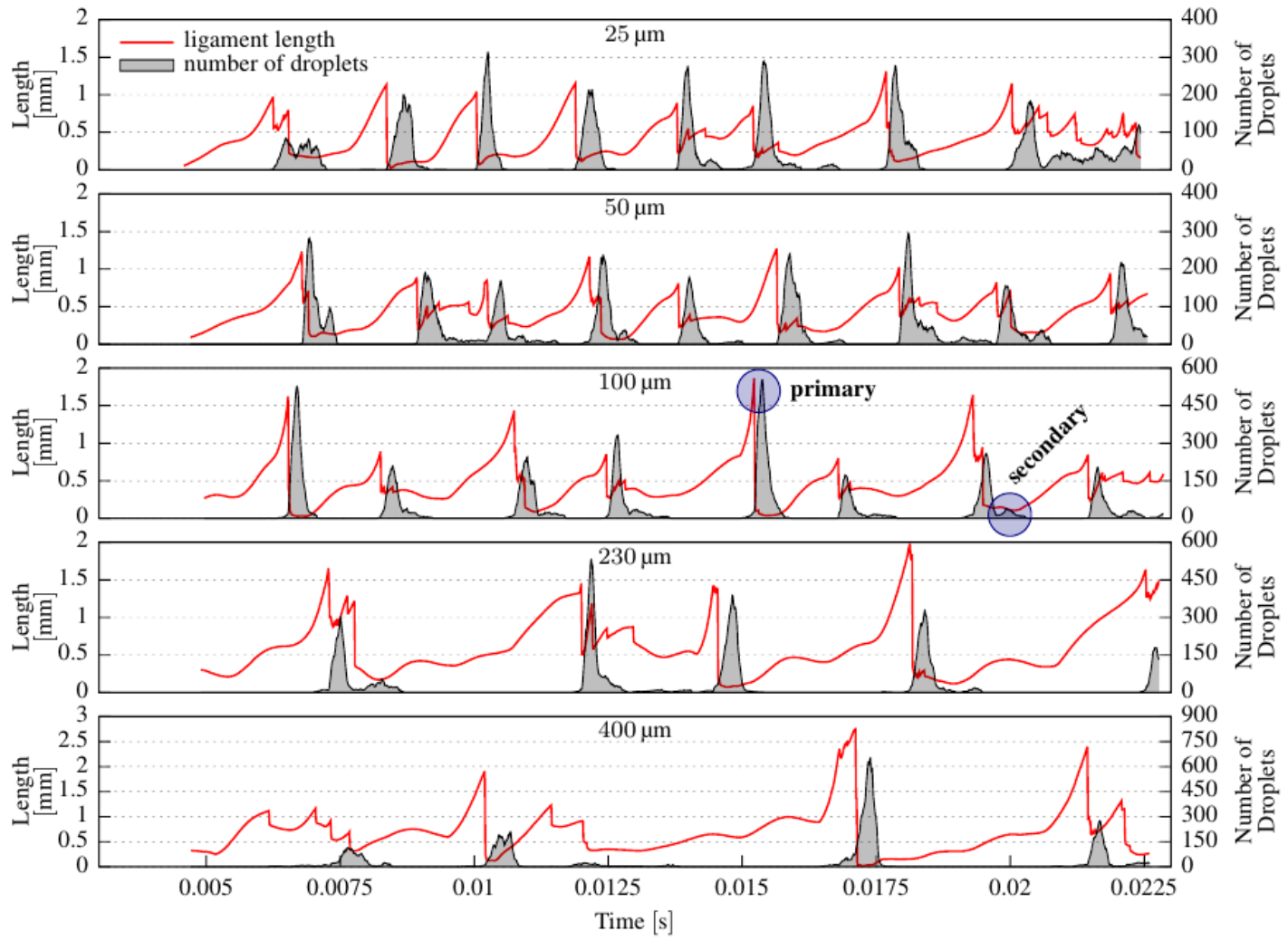

Figure 10. Time series of ligament elongation and droplet formation

almost equal and the temporal evolution of the breakup shows a regular periodic pattern with an almost identical frequency. At a higher trailing edge thickness, the breakup process seems to loose the regularity and strict periodicity. The $100 \mu \mathrm{m}$ case shows more or less a kind of a bimodal behavior of the breakup length. Finally, in case of $230 \mu \mathrm{m}$ and $400 \mu \mathrm{m}$ edge thickness, no regular periodic behavior can be recognized within the simulated period of time.

\subsection{Time series of spray characteristics}

As described in sec. 4 another objective of the post processing was to determine the characteristic volume quantiles of the generated spray. A time series of the volumetric diameters $D_{\mathrm{V} 0.1}, D_{\mathrm{V} 0.5}$ and $D_{\mathrm{V} 0.9}$ is plotted together with the number of droplets in Fig. 11, The curves representing the number of droplets have been added as reference to indicate the break up event.

The graphs of the volumetric diameters $D_{\mathrm{V} 0.1}, D_{\mathrm{V} 0.5}$ and $D_{\mathrm{V} 0.9}$ are characterized by shark fin shaped sections and by partially staggered plateaus. The peaks of the shark fin shaped sections indicate the tear-off of a ligament. These elongated, non-spherical ligaments contain the major part of the liquid volume. The descending part of the curve indicates, that these ligaments are subject to further breakup. This can also be seen in the course of the curve representing the droplet number, where the peaks are located approximately at the center of the descending section of the curves representing the droplet diameters.

The plateau shaped sections indicate, that the breakup process is almost finished. The atomized liquid inside the computational domain is represented by stable size of the droplets. As most of the small droplets are quickly advected out of the domain, the few large droplets which are still present in the computational domain dominate the droplet size distribution at this instance of time, and thus the height of the plateaus. It is important to note that these larger droplets are overrepresented in the graphs, because of their longer residence time compared to smaller droplets. This effect is well known also from laser diagnostics of sprays and called 'biasing'.

Comparing the characteristic diameters resulting from the different trailing edge thicknesses, the $25 \mu \mathrm{m}$ and $50 \mu \mathrm{m}$ case are, again, similar to each other. The shape of the graphs as well as the magnitudes of the characteristic diameters coincide well and show a regular periodic pattern. For increasing trailing edge thicknesses, the magnitudes of the characteristic diameters show a larger spreading and the plateaus are more dominant.

Due to the limited statistics of the numerical data, the derivation of time averaged mean diameters, e.g. the over-all Sauter mean diameter, may be critical and be prone to miscalculation due to the biasing effect. More- 


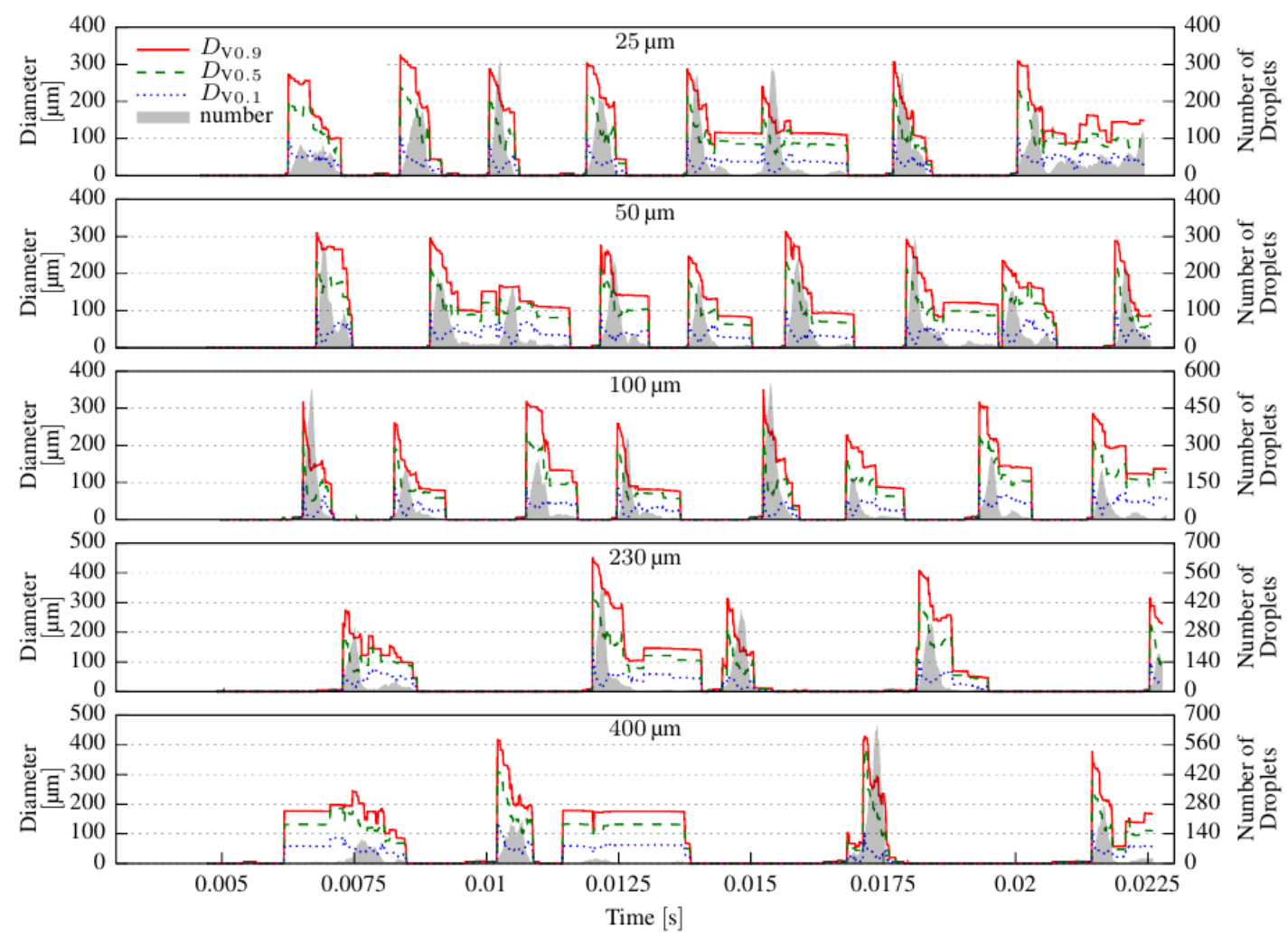

Figure 11. Time series of the spray statistics

over, because of the presence of highly distorted ligaments inside the computational domain, which may be subject to further secondary atomization downstream the actual computational domain, the actual droplet sizing may be incorrect. Therefore, a direct identification of the effect that a thicker trailing edge leads to larger droplets is not possible based on the data of the present study. However, a qualitative comparison of the volumetric diameters $D_{\mathrm{V} 0.1}, D_{\mathrm{V} 0.5}$ and $D_{\mathrm{V} 0.9}$ in Fig. 11 does support the experimental finding that a thicker trailing edge leads to larger droplet size.

\subsection{Effect of trailing edge thickness}

Despite the limited statistics and the fact that the detachment of the ligaments is not perfectly periodic for the cases of the thick trailing edge, arithmetically averaged breakup lengths and breakup frequencies have been extracted from the numerical data. These quantities are plotted in Fig. 12 over the thickness of the trailing edge. The curves show a clear trend as long as the trailing edge is thicker than $100 \mu \mathrm{m}$. Obviously, the break up length increases almost linearly with the thickness of the trailing edge, whereas the break up frequency decays with $\left(1 / h_{E d g e}\right)^{n}$, with an arbitrary parameter $n$. The decay of the break up frequency is consistent with the experimental results as described by Eq 3 .

On the other hand, at a value below $100 \mu \mathrm{m}$, the plots in Fig. 12 reveal, that no significant effect of the trailing

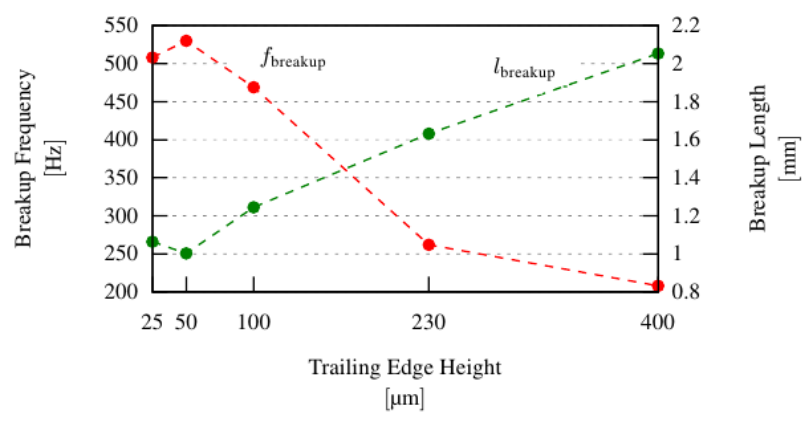

Figure 12. Time series of the spray statistics

edge thickness can be identified. This is in agreement to the experimental results of Inamura et al. [17.

In the experiments a lower limit of break up frequency of approximately $250 \mathrm{~Hz}$ was found for the case of $230 \mu \mathrm{m}$ edge thickness, which is in good agreement with the numerical result of $265 \mathrm{~Hz}$. In this context it is worthwhile to note, that the frequency of the van Karman vortex street of the pure air flow would be $14.1 \mathrm{kHz}$, which is completely different from the break up frequencies observed. Hence, it can be stated that there must be a strong interaction between the air flow and the liquid attached to the trailing edge, that causes such a significant frequency shift. 


\section{CONCLUSIONS}

1. The Smoothed Particle Hydrodynamics method has been proven to be suitable for predicting air blast atomization. In particular, the SPH approach seems to be capable to give insight into details of the liquid breakup process which are not accessible in the experiment.

2. The present $2 \mathrm{D}$ predictions reveal that time series representing the ligament elongation, droplet formation and spray statistics can be extracted by post processing the numerical data. These data may serve to derive simplified models based on correlations for handling air blast atomization. The present predictions do not cover sufficient physical time which is required to extract data that could be cast into meaningful correlations.

3. The effect of the thickness of the trailing edge could be analyzed in terms of break up frequency and break up length. The numerically determined break up frequency matches well to the experimental results.

4. The numerical results confirmed that there are obviously 2 regimes: Above an edge thickness of $100 \mu \mathrm{m}$, the liquid break up depends on the thickness of the edge, below $100 \mu \mathrm{m}$ this is not the case.

5. The numerical results can explain the mechanism which leads to an increase of the droplet size with increasing thickness of the trailing edge, as it was found in the experiment: The accumulation of the liquid at the end of the trailing edge is increasing if the thickness increases. In consequence, the diameter of the generated droplets will increase, and the frequency of the droplet generation bursts decreases.

6. As an outlook it can be stated that high fidelity 3D simulations of the atomizing process will require at least $10^{9}$ particles, and thus adequate computational resources. Our recent studies revealed that such massive computations are feasible with the present SPH code.

\section{ACKNOWLEDGMENTS}

The current work has been partly financially supported by the European Commission under contract FP7-AAT2010-RTD-1 within the 7th framework project "Fuel Injector Research for Sustainable Transport" (FIRST) and within the Clean Sky Research and Technology Development programme in the project "Development of Reliable Emission and Atomization Models for Combustor Design" (DREAMCODE).

This numerical computations were performed on the high performance super computing cluster ForHLR Phase
$I$ at the Karlsruhe Institute of Technology (KIT), Karlsruhe, Germany. The computer cluster was funded by the Ministry of Science, Research and the Arts BadenWürttemberg and the DFG ("Deutsche Forschungsgemeinschaft") within the framework program bwHPC.

\section{REFERENCES}

[1] Th. Sattelmayer and S. Wittig. Internal flow effects in prefilming airblast atomizers: mechanisms of atomization and droplet spectra. Journal of engineering for gas turbines and power, 108(3):465-472, 1986.

[2] A. H. Lefebvre. Airblast atomization. Progress in Energy and Combustion Sience, 6:233-261, 1980.

[3] A. H. Lefebvre. Properties of sprays. Particle \& Particle Systems Characterization, 6:176-186, 1989.

[4] R.A. Gingold and J.J. Monaghan. Smoothed Particle Hydrodynamics: Theory and Application to Non-Spherical Stars. Monthly notices of the royal astronomical society, 181(3):375-389, 1977.

[5] M. Herrmann. On simulating primary atomization using the refined level set grid method. Atomiation and Sprays, 21(4):283-301, 2011.

[6] S. Gepperth, A. Müller, R. Koch, and H.-J. Bauer. Ligament and droplet characteristics in prefilming airblast atomization. In ICLASS, 12th Triennial International Annual Conference on Liquid Atomization and Spray Systems, Heidelberg, Germany, September 2012.

[7] S. Gepperth, R. Koch, and H.-J. Bauer. Analysis and comparison of primary droplet characteristics in the near field of a prefilming airblast atomizer. In Proceedings of ASME Turbo Expo 2013: Turbine Technical Conference and Exposition, number GT2013-94033, San Antonio, Texas, June 2013.

[8] S. Gepperth, E. Bärow, R. Koch, and H.-J. Bauer. Primary atomization of prefilming airblast nozzles: Experimental studies using advanced image processing techniques. In ILASS Europe, 26th Annual Conference on Liquid Atomization and Spray Systems, Bremen, Germany, September 2014.

[9] I. Federico, S. Marrone, A. Colagrossi, F. Aristodemo, and P. Veltri. Simulating free-surface channel flows through SPH. In Proc. 5th international SPHERIC workshop, Manchester, 2010.

[10] C. Höfler, S. Braun, R. Koch, and H.-J. Bauer. Multiphase Flow Simulations Using the Meshfree Smoothed Particle Hydrodynamics Method. In ICLASS, 12th Triennial International Annual Conference on Liquid Atomization and Spray Systems, Heidelberg, Germany, 2012.

[11] S. Braun, L. Wieth, R. Koch, and H.-J. Bauer. A Framework for Permeable Boundary Conditions in 
SPH: Inlet, Outlet, Periodicity. In Proc. 10th international SPHERIC workshop, Parma, Italy, 2015.

[12] J. U. Brackbill, D. B. Kothe, and C. Zemach. A continuum method for modeling surface tension. Journal of computational physics, 100(2):335-354, 1992.

[13] S. Adami, X.Y. Hu, and N.A. Adams. A new surface-tension formulation for multi-phase SPH using a reproducing divergence approximation. Journal of Computational Physics, 229(13):5011-5021, 2010.

[14] L. Wieth, S. Braun, R. Koch, and H.-J. Bauer. Modeling of liquid-wall interaction using the meshless Smoothed Particle Hydrodynamics (SPH) method. In ILASS Europe, 26th Annual Conference on Liquid Atomization and Spray Systems, Bremen, Germany, September 2014.

[15] X.Y. Hu and N.A. Adams. A multi-phase SPH method for macroscopic and mesoscopic flows. Journal of Computational Physics, 213(2):844-861, 2006.

[16] S. Gepperth, D. Guildenbecher, R. Koch, and H.-J. Bauer. Pre-filming primary atomization: Experiments and modeling. In ILASS Europe, 23rd Annual Conference on Liquid Atomization and Spray Systems, Brno, Czech Rep., September 2010.

[17] T. Inamura, M. Shirota, M. Tsushima, M. Kato, S. Hamajima, and A. Sato. Spray characteristics of prefilming type of airblast atomizer. In 12th ICLASS, 2012.

[18] M. Shirota. Private communication, 2013.

[19] S. Braun, M. Krug, L. Wieth, C. Höfler, R. Koch, and H.-J. Bauer. Simulation of primary atomization: Assessment of the smoothed particle hydrodynamics (SPH) method. In ICLASS 2015, 13th Triennial International Conference on Liquid Atomization and Spray Systems, Taiwan, 2015.

[20] S. Gepperth, R. Koch, and H.-J. Bauer. Analysis and comparison of primary droplet characteristics in the near field of a prefilming airblast atomizer. In ASME Turbo Expo 2013: Turbine Technical Conference and Exposition. American Society of Mechanical Engineers, 2013. 